NASA TI X $X=70681$

HIGH ENERGY GAMMA RAY ASTRONOMY

(NASA-TM-X-70681) HIGH ENERGY GAMGA RAY
ASTRCNOAY (NASA) -39 HC $\$ 5.00$ CSCL C3A

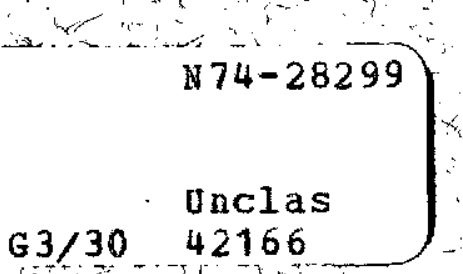

\title{
C. E. FICHTEL
}

\section{JUNE 1974}

GODDARD SPACE FLIGHT CENTER GREENBELT, MARYLAND

Committee on Space Research ZVII Meeting Sao Páulo, Brazit paper IIIA. 1.1. 


\section{HIGH ENERGY GAMMA RAY ASTRONOMY \\ C.E. Fichtel \\ NASA/Goddard Space F1ight Center Greenbelt, Maryland 20771}

\section{Abstract}

From the study of the intensity of the high energy gamma radiation as a function of galactic longitude, it is already clear that cosmic rays are almost certainly not uniformly distributed in the galaxy and are not concentrated in the center of the galaxy. The galactic cosmic rays, and accordingly the magnetic fields to which they are tied, appear to be correlated with galactic structural features, presumably by the gravitational attraction of the matter in the arm segments and the clouds. A strong excess of high energy gamma rays has been observed from the Vela region. If this excess is attributed to cosmic rays released from the vela supernova interacting with the interstellax matter in that region, then on the order of $3.10^{50}$ ergs would have been released by that supernova in the form of cosmic rays. On the extragalactic scale, the diffuse celestial gamma ray flux has both an intensity and an energy spectrum which suggest a possible cosmological origin, although other possible explanations cannot be excluded at this time. The low intensity of the diffuse radiation above $150 \mathrm{MeV}$ also shows that the cosmic rays cannot be universal in a closed universe theory. 


\section{Introduction}

Gamma ray astronomy is now emerging as a very rewarding avenue of astronomical research. Although this very high energy astronomy had long been known to hold potentially great rewards, because of its ability to reveal the dynamic, high energy processes in our galaxy and the universe, its development has been slow and difficult, primarily because of the low intensity of celestial gama rays both in absolute terms and relative to the cosmic rays.

Whereas some gamma radiation presumably arises simply from high energy extensions of the same mechanisms responsible for $\mathrm{X}$-rays (bremsstrahlung, the inverse Compton effect, and magnetobremsstrahlung), other and possibly more significant components probably have origins unique to the $\gamma$-ray region. There are, for examp1e, the gamma rays produced by the decay of neutral pions formed in the collision of energetic cosmic ray nucleons with the interstellar nuclei and by nucleon-antinucleon annihilation. Other processes leading to gamma rays, such as the high energy shock wave possibly assoctated with a supernova burst, are unique to astrophysical bodies.

The first certain detection of celestial gamma rays came from a satellite experiment flown on OSO-III. With this detector Kraushaar et a1. [1], observed the emission of gamma rays with energies above $50 \mathrm{MeV}$ from the galactic disk with a peak intensity toward the galactic center. However, the limited spectral and spatial resolution of this pioneering experiment left many questions 
unanswered. Two second generation spark chamber gamma ray telescopes have now been flown on NASA's SAS-2 and ESRO's TD-1 satellites. Several results from SAS-2 have already been reported in the literature $[2,3,4]$; and, together with the more recent results, also to be summarized here, a more detailed picture of the high energy gamma radiation is beginning to emerge, especially when combined with the data on low energy gamma rays which are becoming available from several balloon and satellite experiments. Results from the TD-1 gamma ray experiment will be presented by Pinkau [5].

Since the work to be reported here draws heavily on the results from the SAS-2 gamma ray experiment, a brief description of that instrument will be given first.

\section{SAS-2 Detector Description}

A schematic diagram of the gamma ray telescope flown on SAS-2. is shown in Fig. 1. The spark chamber assembly consists of 16 spark chamber modules above a set of four central plastic scintillators and another 16 modules below these scintillators. Thin tungsten plates, averaging 0.03 radiation lengths thick, are interleaved between the spark chamber modules, which have an active area of approximately $640 \mathrm{~cm}^{2}$. The large number of thin tungsten plates and spark chambers serve a dual purpose; first, to provide material for the gamma ray to be converted into an electron pair which can then be clearly identified and from which the arrival direction of the gamma ray can be determined, and secondly, to provide a means of determining the energy of the electrons in the pair by measuring the Coulomb scattering. The energy threshold is 
about $30 \mathrm{MeV}$. The energy of the gamma-ray can be measured up to about $200 \mathrm{MeV}$, and the integral flux above $200 \mathrm{MeV}$ can be determined. A more complete discussion of the SAS-2 gamma ray telescope is given by Derdeyn et a1. [6]. The calibration and data analysis are similar to that used for previous balloon gamma ray digttized spark chambers $[7,8,9,10]$. The SAS-2 satel1ite is capable of being pointed in any direction, and normally viewed the same region of the sky for a period of about a week. The orbit is nearly equatorial at an altitude ranging from about $440 \mathrm{~km}$ to $610 \mathrm{~km}$.

\section{Experimental Results and Discussion}

The gamma ray observations which have been obtained with the SAS-2 and other high energy gamma ray experiments divide naturally into four subjects: the strong component from the galactic plane with its hard energy spectrum, the general diffuse radiation, low energy gamma ray bursts, and compact sources. Each of these will now be presented with the experimental results in each case being followed directly by a discussion of their possible interpretation. $3.1 \quad$ Galactic Plane

\subsubsection{Experimenta1 Results}

Relative to the general background celestial diffuse radiation, a strongly enhanced intensity of high energy ( $>30 \mathrm{MeV}$ ) gamma rays is observed along the entire galactic plane. This galactic plane gamma radiation also has a very different energy 
spectrum than the diffuse celestial radiation to be discussed in Section 3.2, the galactic plane spectrum being much flatter and consistent with a predominantly $\pi^{\circ}$ origin above $50 \mathrm{VieV}[3]$. The region in $\ell^{\text {II }}$ from about $330^{\circ}$ to about $40^{\circ}$ is particularly intense, as seen in Fig. 2, which shows the intensity of gamma rays above $100 \mathrm{MeV}$ summed from $b^{I I}=-10^{\circ}$ to $b^{I I}=+10^{\circ}$ and plotted as $a$ function of galactic longitude. Notice particularly that the radiation from the galactic center itself is not more intense than the rest of the interval of about $70^{\circ}$ or $80^{\circ}$ in $l^{I I}$ around the galactic center. This lack of a peak in the gamma ray distribution at the center negates any theory which tries to explain the general enhancement in the region $\left(320^{\circ}<e^{I I}<40^{\circ}\right)$ in terms of a theory involving a strong maximum of emission in the galactic center region.

Fig. 3a $[11]$ shows the angular distribution in $b^{\text {II }}$ of the gamma rays in the interval $335^{\circ}<l^{\text {II }}<25^{\circ}$. The experimental points in this figure have been compared to the sum of two curves with nearly equal areas, one with the detector resolution corresponding to the hard spectrum above $100 \mathrm{MeV}$ and the other a gaussian with a projected $1 \sigma$ of $6^{\circ}$. As will be discussed later, this result implies that the origin of the radiation is about equally divided between close ( $<2$ or 3 kpcs) and more distant regions. In Fig. $3 b$ [11], the distribution $i n b^{I I}$ for that portion of the region $90^{\circ}<\ell^{\text {II }}<270^{\circ}$ for which data exists is plotted, except that the 
Crab region has been excluded. Here there is no narrow peaksuggesting that most of the radiation is coming from relatively close regions as expected since the sun is fairly far from the galactic center and there are no strong sources thought to be near the rim of the galaxy. The other striking feature in Fig. 3 is the very much greater intensity of the galactic radiation in the $335<$ $e^{\text {II }}<25^{\circ}$ region, which was also seen in Fig. 1.

There are two peaks, at $260^{\circ}<\ell^{I I}<270^{\circ}$ and $70^{\circ}<\ell^{\text {II }}<80^{\circ}$, in addition to that due to the Crab nebula $\left(180^{\circ}<\ell^{I I}<190^{\circ}\right)$. The spectra from these regions are also quite hard and indistinguishable from the other regions just mentioned. The region of greater intensity of the two, that between $260^{\circ}$ and $270^{\circ}$, has been discussed by Thompson et al. [4]. This enhancement is centered around $b^{I I}=-3( \pm 1)^{\circ}$ rather than $b^{I I}=0^{\circ}$, and is significant at the $8 \sigma$ leve1. As noted by Thompson et a1. [4] this enhancement could be the result either of a galactic arm feature or a compact source, but the limited extent, the fact it is centered at $b^{I I}=$ $-3( \pm 1)^{\circ}$, and that the Vela $X$ supernova remant is consistent with its location strongly suggest the latter explanation. This possibility will be pursued further in Section 3.4.2.

\subsubsection{Discussion of Galactic Radiation}

The high energy galactic gamma rays are generally thought to result from the interaction of cosmic rays and interstellar matter. This concept is supported by the relatively hard energy spectra observed here for most of the galactic plane. This 
hypothesis will be examined now in terms of some of the models that have been proposed after a brief review of the points in the basic calculation of particular importance here.

The number and energy spectrum of the gamma rays produced by cosmic rays interacting with interstellar matter has been calculated in detail for the case of the cosmic radiation in intergalactic space by several authors (e.g. Stecker [12]; Cava1lo and Gould [13]). The flux of gamma rays with energies greater than $E$ at a distance $r$ is given by the expression

$$
\Phi=\frac{1}{4 \pi} \int \operatorname{Sg}(r, d \theta, d \phi) n(r, d \theta, d \phi) d r d \theta d \phi
$$

where $S$ is the number of gamma rays produced on the average for one interstellar nucleus/sec. and a cosmic ray energy density and spectrum equal to that near the earth, $n$ is the intergalactic nucleon density, and $g$ has been introduced here to represent the ratio of the cosmic ray density to that in the vicinity of the solar system. Following Stecker $[14], S$ is taken to be $1.5 \cdot 10^{-25} / \mathrm{sec}$.

It is worth mentioning at this point that the principal contribution to the high energy gamma radiation from the cosmic ray interactions with interstellar matter comes in the cosmic ray energy range from a few-tenths of a $\mathrm{GeV}$ to a few tens of $\mathrm{GeV}$. Below that energy range the parent $\pi^{\circ}$ mesons are not produced, and at higher energies the contribution is very small because the cosmic ray energy spectrum is decreasing much faster with energy $\left(\mathrm{E}^{\mathrm{I} / 4}\right)$. Hence, when cosmic rays are mentioned here, the energy range mentioned above is implied. 
In the first attempts to compare the observed high-energy gammaray intensity with calculated values, it was assumed (e.g., Kraushaar, et a1. [1]) that the cosmic-ray density was uniform throughout the galaxy so that $g$ could be taken outside the integral in Eq. (1), and was usually set equal to one. Using the $21-\mathrm{cm}$ data to estimate columnar hydrogen density Kraushaar, et al. showed that whereas the calculated intensity was fairly close to that expected in the anticenter direction when the expected intensity was integrated over the solid angle of the detector (which had a gaussian angular sensitivity with a $1 \sigma$ of about $15^{\circ}$ ), the observed intensity in the galactic center region was about four times the calculated value. Thus, the galactic longitudinal dependence was clearly inconsistent with this model, and it could, therefore, not be brought into agreement by assuming a uniformiy higher value of the cosmic-ray density or by assuming that the tota1 matter density was uniformly mach higher beceuse a significant portion of the interstellar hydrogen was in molecular form, for example. More recently, strong, et al. [15], have assumed that the cosmic-ray density has a smooth distribution, but one which increases towards the galactic center according to the equation:

$$
g \propto\left\{\mathrm{Z} \exp \left[-\frac{\mathrm{Z}^{2}}{\mathrm{Z}_{0}}\right] \exp \left(-\frac{\mathrm{R}^{2}}{100}\right)\left[1-\exp \left(-\frac{\mathrm{R}^{2}}{4}\right)\right]\left[1+4 \cos ^{2}(\phi-\phi(\mathrm{R}))\right]\right\}^{\mathrm{n}}
$$

In this relation $\mathrm{Z}$ is the height above the galactic plane, $\mathrm{Z}_{\mathrm{o}}=175 \mathrm{pc}$ and $R=$ distance to galactic center in $k p c$. The choice of this form was based on this expression representing the mean magnetic field $(n=1)$ or the square of the mean magnetic field $(n=2)$, in accordance 
with the work of Thielheim, et a1. [16]. The results were in better agreement with the center-anticenter ratio, but do not agree in detail with more recent SAS-2 results. This work, however; is important as one of the papers breaking with the traditional constant density cosmic-ray concept.

Stecker, et a1. [17], have proposed that the galactic cosmicray flux varies with the radial distance from the galactic center and is about an order of magnitude higher than the local value in a toroidal region between 4 and $5 \mathrm{kpc}$. They further suggest that this enhancement can be plausibly accounted for by Fermi acceleration caused by a hydrodynamic shock driven by the expanding gas in the "3 kpc" arm and invoked in some versions of galactic structure theory. This theory does provide a possible explanation of the general enhancement in the central region as shown in Fig. 4, but possibly not some of the fine details now beginning to appear. There is, or course, also the question of whether or not the Fermi acceleration exists. If it does, then, clearly, the accelerated cosmic-rays could play a role.

In pursuing the problem of galactic gamma radiation, it is important to realize that the one-dimensional fu11-width angular resolution of the high-energy gamma-ray detectors flown thus far has been either several degrees, for StS-2, or about $25^{\circ}$ in the case of OSO-III. Thus, the observed intensity of a feature with a thickness comparable to the disc of the galaxy will decrease approximately as one over the distance once it is more than 2 kps away from SAS-2 
(and closer for OSO-III), and faster if it is also small in extent within the plane. Hence, more distant regions of the galaxy have to be substantia11y more intense than local ones to explain an observed intensity of gamma-rays in any given direction with the present instruments. This consideration, together with the geometrical distribution of the intense high-energy gamma radiation, particularly the broad, relatively flat distribution of the gamma radiation in galactic longitude over $70^{\circ}$ to $90^{\circ}$ in the central region of the galaxy, suggested to Kniffen et a1。[3] and Bignami and Fichtel [18], that the source of the enhancement is possibly predominantly diffuse radiation from the spiral arm segments closest to the sun in the direction of the galactic center.

Bignami and Fichtel [18] have proceeded further and proposed that in general the cosmic-rays are enhanced where the matter is greatest; namely, in the arm segments and clouds. This hypothesis is supported by the following considerations: First, it is assumed that the cosmic-rays and magnetic fields are galactic and not universa1. Then, as shown by Bierman and Davis [19] and Parker [20] in more detail, the magnetic fields and cosmic-rays can only be contained by the weight of the gas through which the magnetic fields penetrate; and, hence, they are tied to the matter. The galactic cosmic-ray energy density cannot substantially exceed that of the magnetic fields, or the cosmic-ray pressure will push a bulge into the fields ultimately allowing the cosmic-rays to escape. The local energy density of the cosmic-rays, however, is about the same as the esti- 
mated energy density of the average magnetic fields and the kinetic motion of matter. Together the total pressure of these three effects is estimated to be equal to the maximum that the gravitational attraction can hold. This feature suggests that the cosmic ray density may generally approach the limit the magnetic fields can contain. This concept is also given some theoretical support by the expected slow diffusion rate of cosmic-rays in the magnetic fields of the galaxy and the very possible high production rate of cosmic-rays, which together also suggest that in general the cosmic rays should be plentiful in a given region and should not move quickly to less dense regions. Therefore, it was assumed that the energy density of the cosmic rays is at or near its saturation value. As a trial assumption, Bignami and Fichtel [18] let the cosmic-ray density be proportional to the matter density. The fluctuations in matter density are then quite important in determining the expected gamma-ray intensity calculated by $\mathrm{Eq}$. (1), since the gamma radiation becomes proportiona1 to $\mathrm{n}^{2}$.

The density distribution of interstellar matter has generally been estimated from $21-\mathrm{cm}$ radio data which, however, indicated only where the atomic hydrogen is and not the ionized and molecular hydrogen. There are in addition some problems associated with the direct interpretation of the $21 \mathrm{~m}$ data as discussed, for example, by Simonson [21]. First, there is clearly significant absorption of the $21-\mathrm{cm}$ line over a band in galactic longitude about the galactic center, and also there are indications of high optical depth along 
spiral arm segments. Second, the interpretation of the observed intensity in the $21-\mathrm{cm}$ line in terms of density depends on the assumed galactic velocity field, and there is increasing reason to believe the velocity pattern is not as simple as assumed in the earliest models. It is actually this latter problem which is of greater concern here, because it affects the peak-valley ratio of the matter density distribution.

It seems plausible, relying again both on measurements from external galaxies and on the density wave theory for the spiral pattern (e.g., Roberts and Yuan [22]), to assume at least for the inner galactic arms that this ratio is five to one. With this assumption, the center to anticenter ratio can be explained as well as the distribution in the $310^{\circ}<\ell^{I I}<50^{\circ}$ interval in the genera1 way permitted by a cylindrical model approximation as shown in Fig. 5 . In this model the Sagitarius arm makes a major contribution, and it is close enough in the $l^{I I}=0^{\circ}$ direction that its width in $b_{\text {II }}$ is less than the detector resolution. Fig。 3, as noted earlier, clearly shows a distribution of at least two components.

In Fig. 2, the sharp decrease at about $\ell^{I I}=50^{\circ}$ is consistent with the tangent to the Sagitarius arm as shown in Fig. 6. The valley from $50^{\circ}$ to $70^{\circ}$ is consistent with the lack of features in that direction and the increase from $70^{\circ}$ to $80^{\circ}$, although of marginal staatistical significance, is consistent with the position of the Orion arm. The lesser decrease at $330^{\circ}$ corresponds possibly to the tangent to the Norma arm, but with there still being contributions 
from the Scutum and Sagitarius arms which are drawn out further on that side of the galaxy.

3.2. Diffuse High Energy Gamma Radiation

3.2.1. Experimental Data

The gamma-ray experiment on OSO-III of Kraushaar et a1. [1] first observed a finite, apparently constant diffuse flux for regions of the sky away from the galactic plane. An integral value of $(3.0 \pm 0.9) \cdot 10^{-5} /\left(\mathrm{cm}^{2}\right.$ sterad。 $\left.\mathrm{sec}_{0}\right)$ was quoted for the intensity above $100 \mathrm{MeV}$, but essentially no energy spectra1 information was obtained. SAS-2 has now also observed a finite, diffuse flux of gamma-rays with a steep energy spectrum in the energy region from 35 to $170 \mathrm{MeV}$ in severa1 regions with $\left|b^{I I}\right|>30^{\circ}$ (Fichte1 et a1。 $[2,11]$. The spectrum is shown in Fig. 7. Representing the energy spectrum by a power law of the form $\mathrm{dJ} / \mathrm{dE}=\mathrm{AE}^{-\alpha}$ over this energy range, $\alpha$ is found to be $2.5+0.4$. Combining this result with existing low-energy gamma-ray data yields an energy spectrum which is not a simple power law in energy, as in the X-ray region, but which demonstrates first an increase and then a decrease in slope, as shown in Fig. 7.

\subsubsection{Discussion of Diffuse Radiation}

If it is to be assumed that cosmic-rays pervade the entire universe, a specific cosmological model must be selected before any conclusions can be drawn. However, the relatively low intensity observed in the 100 to $170 \mathrm{MeV}$ region places constraints on the distance to which cosmic rays at the density observed in the vicinity of the earth may extend in a closed universe. Since the limit will 
be seen to be sufficiently close in distance to avoid major cosmological effects, the calculation is straightforward. Using the gamma-ray measurements mentioned in the last paragraph the limiting radius is about fifty mega-parsecs for an interstellar density required for a closed universe, name $1 \mathrm{y}$ about $10^{-5} / \mathrm{cm}^{3}$. Thus, a cosmic-ray density equal to that near the earth cannot pervade the universe, if it is closed, but the possibility that cosmic-rays at the loca1 density exist throughout our local super-cluster of galaxies cannot be eliminated. Future gamma-ray observations at higher energies could further restrict this limit, unless, of course, a $\pi^{\circ}$-like spectrum is seen at these higher energies instead of the steep spectrum seen at 30 to approximately $170 \mathrm{MeV}$. If the universe is not closed, the matter density may be much lower. In that case specific cosmological models must be considered because the large distances require careful consideration of the appropriate red shifts.

It is also instructive to consider the possible origin of the diffuse radiation, since at least one explanation relates to primordial cosmic-rays. First of a11, there is the possibility that the diffuse radiation is the sum of many weak discrete or extended sources of unknown origin. Only future experimental results can clarify the picture with regard to that possibility. There are, however, at least two other possibilities; one that the radiation comes from diffuse electrons interacting with matter, photons, or magnetic fields, and the other is that the gamma rays are of cosmological origin. 
With regard to the diffuse electron possibility, bremsstrahlung seems unlikely. In an energy region, 1 to $10 \mathrm{MeV}$, where an increased slope would be expected due to an increasing rate of energy loss, the opposite is observed. For both synchrotron and Compton radiation, the observed photon spectrum would imply a similarly-shaped parent electron spectrum which would have even very much sharper spectral features. Further, for al1 three cases, the intensity seems high to be consistent with reasonable estimates of the interstellar parameters.

Of the pure gamma-ray cosmological hypotheses, there are at least three that seem to be possible candidates. They are the cosmic-rayinterstellar matter interaction model, the particle-antiparticle annihilation in the baryon symmetry steady-state mode1, and the cosmicray-blackbody interaction model. In all theories, the resulting gammaray spectrum is red-shifted substantially by the expansion of the universe.

In an expanding mode1 of the universe, the density of matter is much greater in the cosmological past than it is observed to be in the present. However, since the gamma radiation produced in interactions of cosmic-rays with matter in the distant past reaches us from large distances, the energy of these photons is degraded by the cosmological redshift caused by the expansion of the universe. One curve developed by Stecker [31] involving red-shifts up to about 100 is shown in Fig. 7. The theoretical curve is seen to agree with experimental data reasonably we11. If the maximum red-shift is at least 50 , as the data implies, then the density of cosmic rays in intergalactice space is 
$10^{-4}$ of the local galactic value for an intergalactic matter density of $10^{-5} / \mathrm{cm}^{3}$ 。 This model does imply what appears to be an implausibly high cosmic ray energy density at early times in the universe.

An alternate attempt to explain the gamma radiation through redshifted gamma rays from $\pi^{\circ}$ decay arises from the big bang theory of cosmology with the principle of baryon-symmetry。 Harrison [32] was one of the first to propose a model of this type. Omnes [33] following Gamow [34], considered a big-bang mode1 in which the universe is initially at a very high temperature and density, and then shows that, if the universe is baryon-symmetric, a separation of matter from antimatter occurred at $\mathrm{T}>30 \mathrm{MeV}$. The initial phase separation of matter and anti-matter leads ultimately to regions of pure matter and pure anti-matter of the size of galactic clusters, Stecker, Morgan, and Bredekamp [35] have predicted the gamma ray spectrum which would be expected from annihilation at the boundaries of such clusters from the beginning of their existence to the present. This spectrum is very similar (essentially indistinguishable) to the one in Fig. 8 in the energy range for which data exists, and is not included in the figure for that reason. The final model involves cosmic ray interactions with the blackbody radiation at an early point in cosmological time. Wolfendale [36] has shown that this theory is also a possibility.

\subsection{Low Energy Gamma Ray Bursts}

In 1973 , Klebesadel et al。 [37] reported the detection of low energy gamma ray bursts. These have subsequently been confirmed by other groups. The most recent catalog of these hard X-ray--soft gamma 
ray bursts is that of Strong et al. [38]. The SAS-2 anticoincidence dome provides a very large detector for the high energy portion $(\gg 0.3 \mathrm{MeV})$ of these events and for completeness the observations of SAS-2 will be reported here, even though they fall in the low energy gamma ray category. From any side or the top of the SAS-2 experiment, there is an effective area of about $2.5 \cdot 10^{3} \mathrm{~cm}^{2}$. The energy threshold varies with position over the $2 \mathrm{~cm}$ thick dome and with incident angle at any one point. However, except in the vicinity of the bottom rim its response is fairly uniform. Averaging over the dome it is found, that the effective threshold for detection is about $0.15 \mathrm{MeV}$, the efficiency rises to about $4 \%$ at $0.2 \mathrm{MeV}, 15 \%$ at $0.4 \mathrm{MeV}$, and $20 \%$ at $0.6 \mathrm{MeV}$. The counting rate of the anticoincidence dome when there is no increase due to the trapped radiation in the Atlantic anomaly is about $4.2 \cdot 10^{3} \mathrm{cts} / \mathrm{sec}$, and it remains quite steady。

During the period of its operation, November 20, 1973 through June 8,1974, SAS-2 [11] dẹtected two events observed by other satellites at 23:27:53 on March 2, $1973[39,40]$ and $07: 07: 28$ on June 6,1973 [40] and discovered one at $18: 47: 08$ on June 6,1973 which was later confirmed by other satellites $[39,40]$. In addition, two events on the list of those detected by other satellites at $20: 27: 39$ on December $18,1972[39,40,41]$ and $08: 04: 32$ on May 7,1973 [38] were not seen by SAS-2. In view of the high sensitivity of SAS-2, these negative results suggest that either the earth occulted the source, as it w11l about $40 \%$ of the time on the average, or the energy spectrum of the events was very steep and there was no significant flux about $0.2 \mathrm{MeV}$. 
It is interesting that two of the three events seen by SAS-2. occurred on the same day, 6 June, 1974. The time profiles of these two events are shown in Fig. 8. There were, in fact, only three detected cases in the approximately $61 / 2$ months that the A-dome rate exceeded threshold leve 1 for more than 3 readout intervals (approximate1y 2.3 seconds), except when the satellite passed through the Atlantic trapped radiation anomaly. Hence, the second June 6 event immediately attracted interest as a possible burst event, and it was subsequently confirmed by the IMP-7 satellite (C1ine and Desai [40]) and the Vela system (Klebesaded, et a1. [39]. Notice that for the June 6 event which the Vela trigger system did detect, the Vela time for first detection occurs after the event has started and roached a leve 1 almost an order of magnitude above the SAS-2 threshold. This situation is also true for the March 2, 1973 event.

In spite of the apparently significantly greater sensitivity of SAS-2 as suggested by these comparisons and direct calculations, no other events of more than two seconds were observed beyond the three mentioned. A more detailed discussion of these events is given by Fichtel et al. [11]

\subsection{Compact Sources}

Being above the atmosphere, the ability of the SAS-2 gamma ray telescope to see compact or point sources of gamma rays has been limited only by the diffuse celestial and galactic background and its own size and collection time. Desired quality aspect data for SAS -2 has only recently been received. It has now been applied to 
the entire galactic plane and two sources seen in the galactic plane will be discussed here.

\subsubsection{Crab Nebula}

There have now been a large number of positive results reported for gamma radiation from the Crab nebula $[42,43-51]$. Whereas many are of marginal statistical weight, there is a general consistency of the data and the positive flux reported from the SAS-2 data [42] was a more than $8 \sigma$ effect. Within the limit of angular resolution of the detectors the source is a point source. The flux above $100 \mathrm{MeV}$ is $(4.1 \pm 0.7) \times 10^{-6} / \mathrm{cm}^{2}$ sec., and above $35 \mathrm{MeV}$ it is $(1.9 \pm 0.3) \times 10^{-5} /$ $\mathrm{cm}^{2}$ sec [42]. Most of the results in the gamma ray region refer only to the pulsed component, since the majority of the results are from balloon data and the atmospheric background is sufficiently large so that only a pulsed flux is detectable, in general, for intensity levels as low as that from the Crab nebula. The SAS-2 data taken together with the balloon results do suggest that the pulsed component plays a bigger role in the gamm ray region than in the X-ray region. The observed spectral distribution present in Fig. 9 is seen to be essentially a power law and inconsistent with a $\pi^{\circ}$-decay spectrum. The most likely emission mechanism is electro-magnetic emission by energetic electrons accelerated by the pulsar, but more precise results are needed to allow a detailed investigation of specific mode1s. 


\subsubsection{Vela Region}

The large intensity increase in the Vela supernova remnant direction was mentioned earlier and has been discussed in a previous paper (Thompson et a1. [4]). This enhancement is significant at above the $8 \sigma$ level. The energy spectrum is hard and conststent with a predominantly $\pi^{\circ}$ origin. Further, an extrapolation of the $\mathrm{X}$-ray spectrum $[52,53,54,56]$ to the high energy gamma ray region lies well below the results presented here, indicating that some different production mechanism is present at high energies.

Assuming the excess gamma radiation to be due to cosmic rays association with the Vela supernova, sssuming the supernova remant to be 460 parsecs away, and assuming the matter density to be about 1.5 protons $/ \mathrm{cm}^{3}, 3 \cdot 10^{50}$ ergs of energy would be in the form of cosmic rays from this supernova. This is a number in the energy range, $10^{49}$ to $10^{51}$ ergs, needed if supernovae are to be the main source of galactic cosmic rays and is also in the range predicted by Colgate [52] for the supernova hydrodynamic shock theory, as noted by Thompson, et a1. [4].

It is interesting to note that if a similar amount of energy were in the form of cosmic rays around the crab nebula, the intensity would still be only about $1 / 15$ th of the Crab flux since the Crab is approximately four times as far away from the sun as Vela.

\subsection{Summary}

As the previous sections have indicated, although celestial high energy gamma-ray research is just emerging as the newest branch of 
astronomy, it is already providing results which are of considerable importance in the study of the galaxy and the universe. It was seen that cosmic rays are almost certainly not uniformly distributed in the galaxy and are not concentrated in the center of the galaxy. The galactic cosmic-rays are more probably tied to structural features by magnetic fields, which are in turn held by the matter in the arm segments and clouds. However, the detailed study of the dynamic influence of the cosmic rays in source regions and the study of their diffusion in the galaxy will have to wait for a gamma-ray telescope an order of magnitude or more sensitive than SAS-2 and one with somewhat better angular resolution even than SAS-2.

A strong excess of high energy gamma rays was observed from the Vela region. If this excess is attributed to cosmic rays released from the Vela supernova interacting with the interstellar matter in that region, as seems reasonable, then on the order of $3.10^{50}$ ergs would have been released by that supernova in the form of cosmic rays. On an extragalactic scale, it has been seen that it is possible to say that the cosmic ray density seen near the earth is not universal if the universe is closed; at present it is not possible, on the basis of the diffuse ganma-ray data, to exclude the possibility that the cosmic rays pervade the local supercluster. However, the apparent non-uniform distribution of cosmic-rays in the galaxy, if firmly established, would be a difficulty for this latter concept. 
The diffuse celestial gamma-ray spectrum that is observed presents the interesting possibility of cosmological studies and possible evidence for a residual universal cosmic-ray density or even more interestingly support for galactic superclusters of matter and antimatter remaining from a baryon symmetric big bang. Again, a future gamma-ray instrument of much larger sensitivity with modest energy and angular resolution can answer these questions. 
REFERENCES

[1] W. L. KRAUSHAAR, G. W. CLARK, G. P. GARMIRE, R. BORKEN, P. HIGBIE, V. LEONG, and T. THORSOS, Astrophys. J. 177, 341 (1972).

[2] C. E. FICHTEL, R. C. HARTMAN, and D. A. KNIFFEN, Astrophys. J. (Letters) 186, L99 (1973)。

[3] D. A. KNIFFEN, R. C. HARTMAN, D. J . THOMPSON, and C. E. FICHTEL, Astrophys。 J。 (Letters) 186, L105 (1973).

[4] D. J. THOMPSON, G. F. BIGNAMI, C. E. FICHTEL, and D. A. KNIFFEN, "SAS-2 Observations of the High Energy Gamma Radiation from the Vela Region," to appear in Astrophys J. (Letters) in June (1974).

[5] K. PINKAU, this COSPAR Conference (1974).

[6] S. M. DERDEYN, C.H. EHRMANN, C. E. FICHTEL, D. A. KNIFFEN, and R. W. ROSS, Nucl. Instrum。 Methods 98, 557 (1972).

[7] C. E. FICHTEL, D. A. KNIFFEN, and H. B. ÖGELMAN, Astrophys. J。 158, 193 (1969).

[8] D. A。 KNIFFEN, NASA Technical Report R-308 (1969).

[9] C. E. FICHTEL, R. C. HARTMAN, D.A. KNIFFEN, and M. SOMMER, Astrophys. J。171, 31 (1972)。

[10] D. J. THOMPSON, "A Study of 30-300 MeV Atmospheric Gamma Rays," Ph.D. Thesis, U. of Maryland (1973).

[11] C. E. FICHTEL, D. A. KNIFFEN, R. C. HARTMAN, D. J. THOMPSON, G. F. BIGNAMI, H. B. OGELMAN, and T. TUMER, "High Energy Gamma Ray Astronomy Results from SAS-2," to be published (1974).

[12] F. W. STECKER, Astrophys, and Space Sci. 6, 377 (1970). 
[13] G. CAVAllo and R. J. GOULD, Nuovo Cim. 2B, 77 (1971).

[14] F.W. STECKER, Astrophys。J。185, 499 (1973).

[15] A.W. STRONG, J. WDOWCZYK, and A. W. WOLFENDALE in: Gamma Ray Astrophysics (Ed.F. W. Stecker and J. I. Trombka), U. S. Government Printing Office, Washington, D.C., P. 259 (1973).

[16] K. O. THIELHEIM, G. WENNER, W. H. STEEB, J. VOSS, and H. J. KUCKOFF, Proc. 12th Int. Confo Cosmic Rays (1971)。

[17] F. W. STECKER, J. L. PUGET, A. W. STRONG, and J. H. BREDEKAMP, Astrophys. J. (Letters) 188 , in press (1974)

[18] G. F. BIGNAMI and C. E. FICHTEL, Astrophys. J. (Letters) 189, L65 (1974)。

[19] I. BIERMAN and L. DAVIS, Z。J. Astrophys. 51, 19 (1960).

[20] E。 N. PARKER, Astrophys. J. 145, 811 (1966).

[21] S. C. SIMONSON, Astron. and Astrophys. 9, 163 (1970).

[22] W. W. ROBERTS and C. YUAN, Astrophys. J. 161, 887 (1970).

[23] S. V. GOLENETSKII, E. P. MAZETS, V. N. ILINSKII, R. L. APTEHAR', M. M. BREDOV, YU. A. GUR'YAN, and V. N. PANOV, Astrophys J. (Letters) 9 , L9 (1971).

[24] G. VEDRENNE, F. ALBERNHE, I. MARTIN, and R. TALON, Astron. and Astrophys. 15, 50 (1971).

[25] H. A. MAYER-HASSELWANDER, E. PFEFFERMANN, K. PINKAU, H. ROTHERMEL, and M. SOMMER, Astrophys。 J. (Letters) 175, L23 (1972).

[26] J. I. TROMBKA, A. E. METZGER, J. R. ARNOLD, J. L. MATTESON, R.C. READY, and L. E. PETERSON, Astrophys. J. 181, 737 (1973). 
[27] G. H. SHARE, R. L. KINZER, and N. SEEMAN, Astrophys。J. 187, $45(1974)$

[28] B. AGRINIER, M. FORICHON, J. P. LERAY, B. PARLIER, T. MONTMERLE, G. BOELIA, L. MARASCHI, B. SACEO, L. SCARSI, J. M. DaCostA, and R. PALMEIRA, 13th Internationa1 Cosmic Ray Conference, Vo1. 1 , 8 (1973)。

[29] F. KUO, G. FRYE, P. ALBATS, G. THOMSON, A. ZYCH, V. HOPPER, and 0 . MACE, 13th International Cosmic Ray Conference, Vol。 1,20 (1973)。

[30] V. D. HOPPER, O.B. MACE, J. A. THOMAS, P. ALBATS, G. M. FRYE, JR., G. B. THOMSON, and J. A. STAIB, Astrophys. J。 (Letters) 186, L55 (1973).

[31] F. W. STECKER, Astrophys. J。 157, 507 (1969)。

[32] E.R. HARRISON, Phys。Rev. Letters 18, 1011 (1967).

[33] R. OMNES, Phys. Rev。Letters 23, 38 (1969)。

[34] G. GAMOW, Phys. Rev. 74, 505 (1948).

[35] F. W. STECKER, D. L. MORGAN, and J. BREDEKAMP, Phys. Rev. (Letters) $\underline{27}, 1469$ (1971).

[36] A. W. WOLFENDALE, "Explanation of the Spectral Shape in the Energy Range $10^{14}-10^{20} \mathrm{eV}, "$ Royal Society Discussion Meeting, Feb. 20-21 (1974)。

[37] R. W. KLEBESADEL, I. B. STRONG, and R. A. OLSON, Astrophys. J. (Letters) 182, L85 (1973).

[38] I. B. STRONG, R. W. KLEBESADAL, and R. A. OLSON, Astrophys. J. (Letters) 188, L1 (1974). 
[39] R. W. KLEBESADEL, R. A. OLSON, and I. B. STRONG, private communication (1974).

[40] T. CLINE and U. DESAI, private communication of work to be published (1974).

[41] W. L. IMHOF, G. H. NAKANO, R. G. JOHNSON, J. R. MILNER, J. B。 REAGAN, R. W. KLEBESADEL and I. B. STRONG, "Spectra Measurements of a Cosmic Ganma Ray Burst with Fast Time Resolution," to be published in Astrophys。 J. (1974).

[42] D. A. KNIFFEN, R. C. HARTMAN, D. J。 THOMPSON, G. F. BIGNAMT, and C. E. FICHTEL, "The Gamma Radiation above $35 \mathrm{MeV}$ From the Crab Nebula," submitted to Nature (1974).

[43] J. Ma RANKIN, private communication (1974)。

[44] G. J. FISHMAN, R. F. HARNDEN, JR,, and R. C. HAYMES, Astrophys. J. (Letters) 156, L17-L10 (1969)。

[45] J. D. KURFESS, Astrophys。 J. (Letters) 169, L39-I42 (1971).

[46] R. BROWNING, D. RAMSDEN, and P. J. WRIGHT, Nature 232, 99-101 (1971)。

[47] G. KETTENRING, H. A MAYER-HASSELWANDER, E. PFEFFERMANN, K. PINKAU, H. ROTHERMEL, and M. SOMMER, 12th International Cosmic Ray Conference 1 , 57-62 (1971).

[48] P. ALBATS, G. M. FRYE, JR., A. D. ZYCH, O. B. MACE, V. D. HOPPER, and J. A. THOMAS, Nature 240, 221-224 (1972).

[49] B。 MCBREEN, S. E. BALL, JR。, M。CAMPBELL, K. GREISEN, and D. KOCH, Astrophys. J. 184, 571-580 (1973). 
[50] B. PARLIER, B. AGRINIER, M. FORICHON, J. P. LERAY, G. BOELIA, L. MARASCHI, R. BUCHERI, N.R. ROBBA, and L. SCARSI, Nature Phys. Science 242, 117-120 (1973)。

[51] H. HELMKEN and J. HOFFMAN, 13th International Cosmic Ray Conference $\underline{1}, 31-35$ (1973).

[52] S. A. Colgate, Can. J. Phys. 46, S476 (1968)。

[53] A. N. BUNNER in: The Gum Nebula and Related Problems, ed. S. P. MARAN, J. C. BRANDT, and T. P. STECHER (GSFC Report $\mathrm{X}-683-71-375)(1971)$

[54] E. KELIOGG, H. TANANBAUM, F。'R. HARNDEN, JR., H. GURSKY, and R. GIACCONI, Astrophys. Jo 183, 935 (1973).

[55] F. R. HARNDEN, JR., W. J. JOHNSON, III, and R. C. HAYMES, Astrophys. J。 (Letters) 172, 191 (1972)。

[56] F. R. HARNDEN, JR, and P. GORENSTEIN, Nature 241, 107 (1973). 


\section{FIGURE CAPTIONS}

Fig. 1 - Schematic Diagram of the SAS-2 Gamma-Ray Experiment [6]. Fig. 2 - Distribution of high-energy (> $100 \mathrm{MeV}$ ) gamma-rays along the galactic plane. The data are the work of kniffen, et a1. [3] and Fichtel et al. [11]. The diffuse background leve1 is shown by a dashed line. The SAS-2 data are summed from $b_{11}=$ -10 to $b_{11}=+10$. The ordinate scale is approximately in units of $10^{4} \times$ photons $/ \mathrm{cm}^{2}$ radian sec.

Fig. 3(a) - Distribution of high-energy $\left(\mathrm{E}_{\gamma}>100 \mathrm{MeV}\right)$ gamma-rays summed from $\ell_{I I}=335^{\circ}$ to $\ell_{I I}=25^{\circ}$ as a function of $b_{I I}$. The solid line represents the sum of two distributions with equal areas, one representing only the detector resolution and the other a gaussian with $\sigma=6^{\circ}$.

(b) - Distribution of high energy $\left(\mathrm{E}_{\gamma}>100 \mathrm{MeV}\right)$ gamma rays summed from $90^{\circ}<l^{\mathrm{II}}<170^{\circ}$ and $200^{\circ}<l^{\mathrm{II}}<270^{\circ}$, where data exists.

Fig. 4 - Comparison of the longitudinal distribution of galactic $y$-radiation observed on SAS-2 with the distribution given by the theoretical model of Stecker, et al. $[17]$.

Fig. 5 - Longitudinal distribution of galactic gamma-flux integrated over $\pm 10^{\circ}$ in $b^{I I}$. SAS-II points are given together with their error bars. The thick line represents the cylindrical symmetry approximation model of Bignami and Fichtel [18] smoothed in $10^{\circ} \ell^{I I}$ intervals. The thin line represents the model in $2^{\circ}$ intervals. The dotted line (--) gives the contribution of 
the Sagittarius and Norma-Scutum arms and dash-dot (...), the contribution of the Sagittarius arm along.

Fig. 6 - A smoothed spatial diagram of the locations of the maxima of the matter density deduced from $21-\mathrm{cm}$ HI 1 ine measurements, and the density-wave theory by Simonson [21].

Fig. 7 - Diffuse celestial radiation observed by several experiments. Also shown are the straight line extrapolation of the $\mathrm{X}$-ray data (solid 1 ine) and the curve predicted by the cosmic-rayintergalactic matter interaction cosmological model with $\mathrm{Z}_{\mathrm{MAX}}=$ 100 (Stecker [23] discussed in the text-dashed line). The points labeled in the Figure refer to Golenetskii et a1. [23], Vedrenne et al. [24], Mayer-Hasselwander et a1. [25], Trombka et a1. [26], Share et a1. [27], Agrinier et a1. [28], Kuo et al. [29], Hopper et al. [30], Fichtel et a1. [2], and Fichtel et al. [11].

Fig. 8 - Time history of two low energy gamma ray bursts observed in the SAS-2 A-Dome on June [1l]. In each case the count rate in the interval prior to the first one shown was less than 250 counts/(.768 second interval) "VELA" refers to the first indication of an event as seen by the VELA low energy gamma ray burst experiment [39].

Fig. 9 - Spectrum observed from the region of the crab nebula. The SAS-2 data [42] are presented together with those of Fishman et a1. [44], Kurfess [45], Browning et a1. [46], Kettenring et a1. [47], Albatts et al. [48], McBreen et a1. [49], Par1ier et a1. [50] and Helmken and Hoffman [51]. 


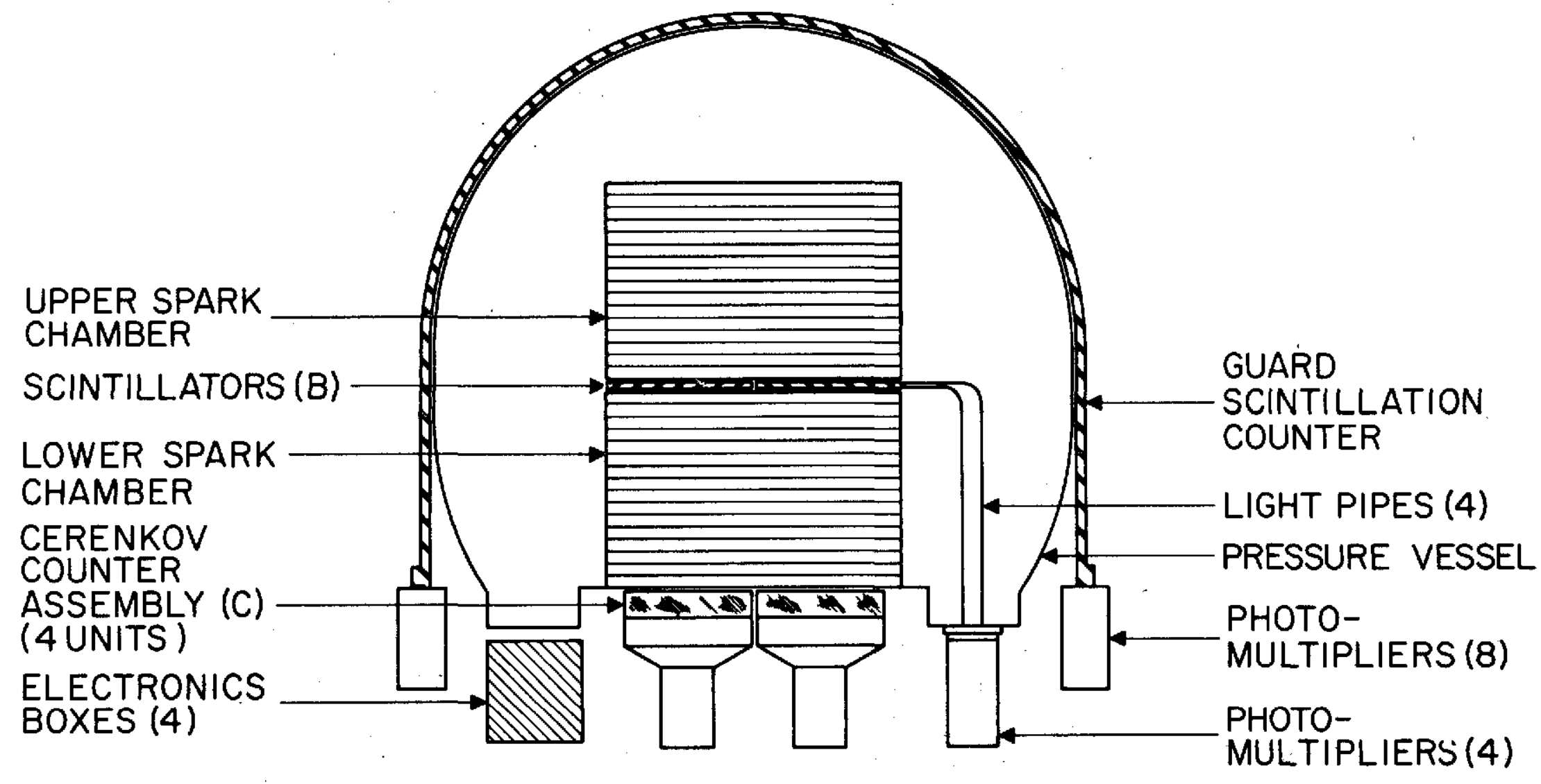

SAS-2 GAMMA RAY EXPERIMENT 


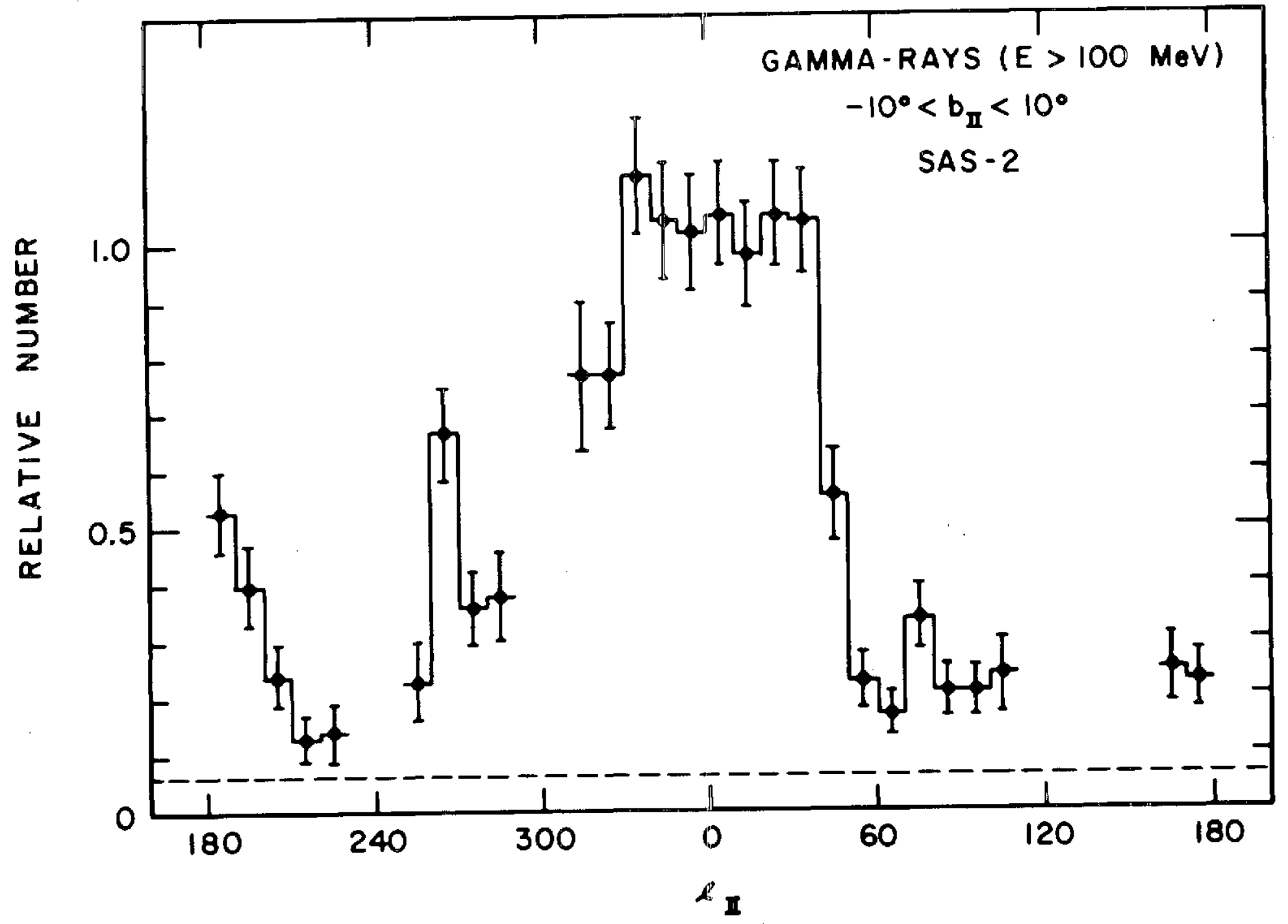

Fig. 2 


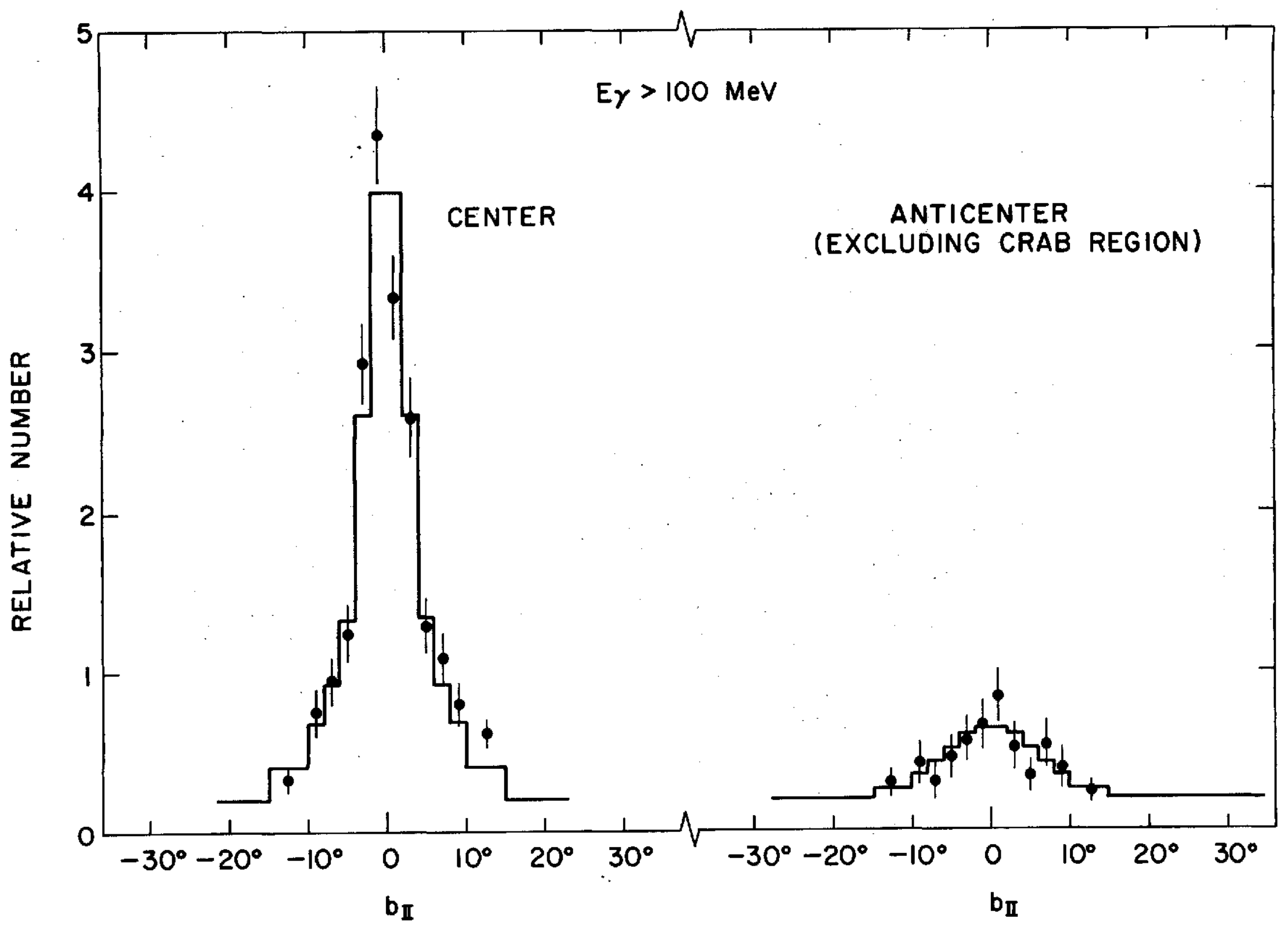

Fig. 3 


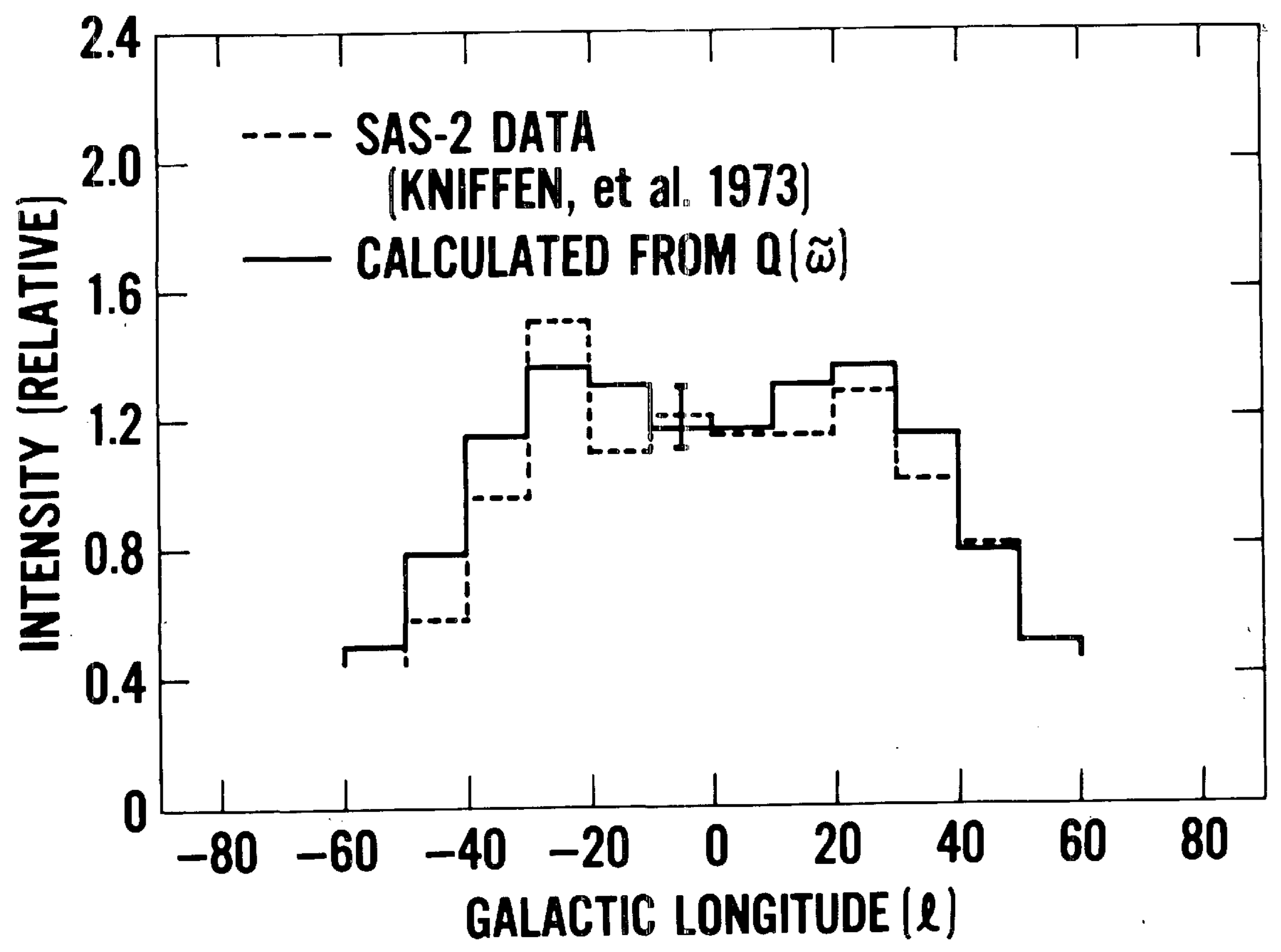

Fi.g. 4 


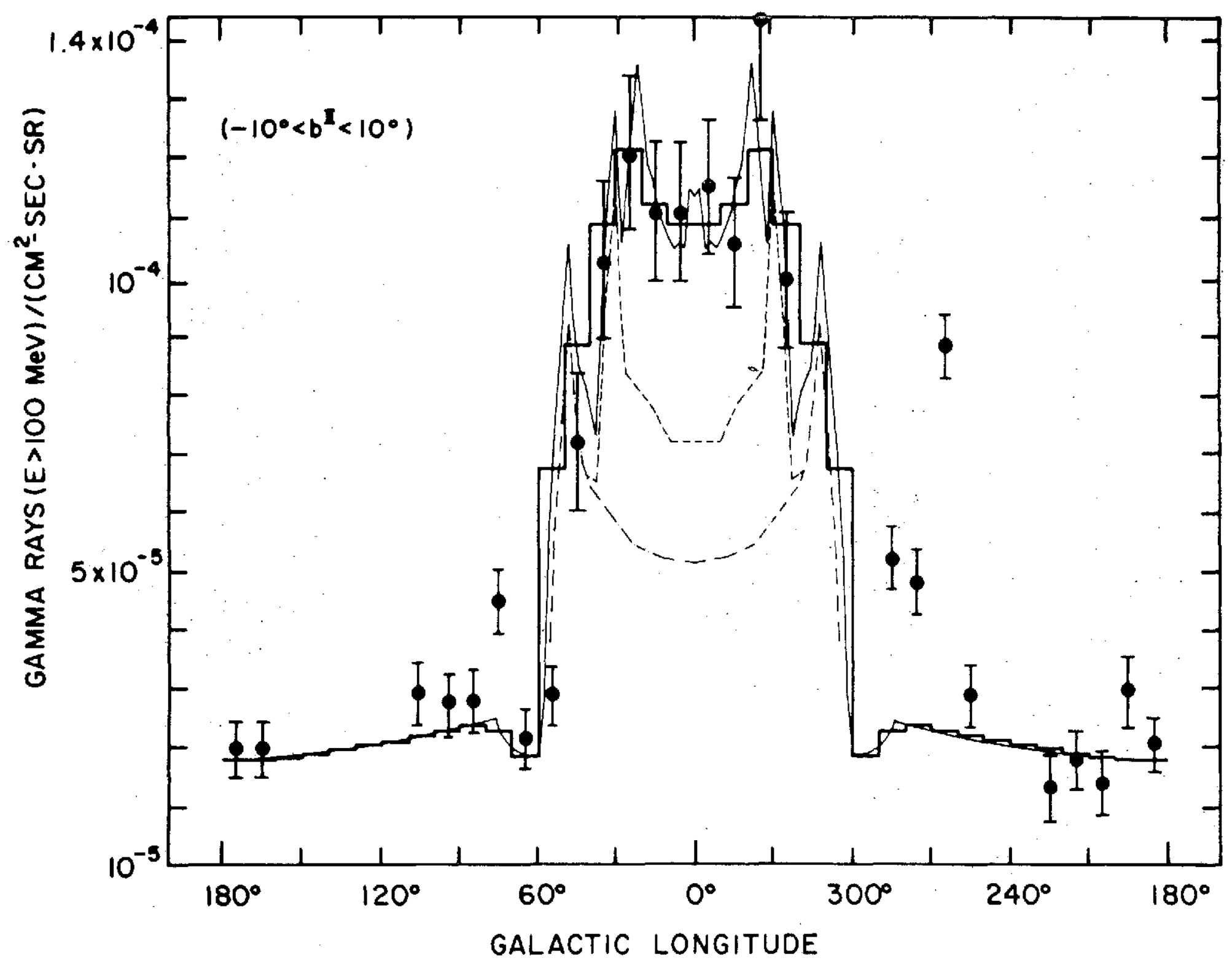

Fig. 5 


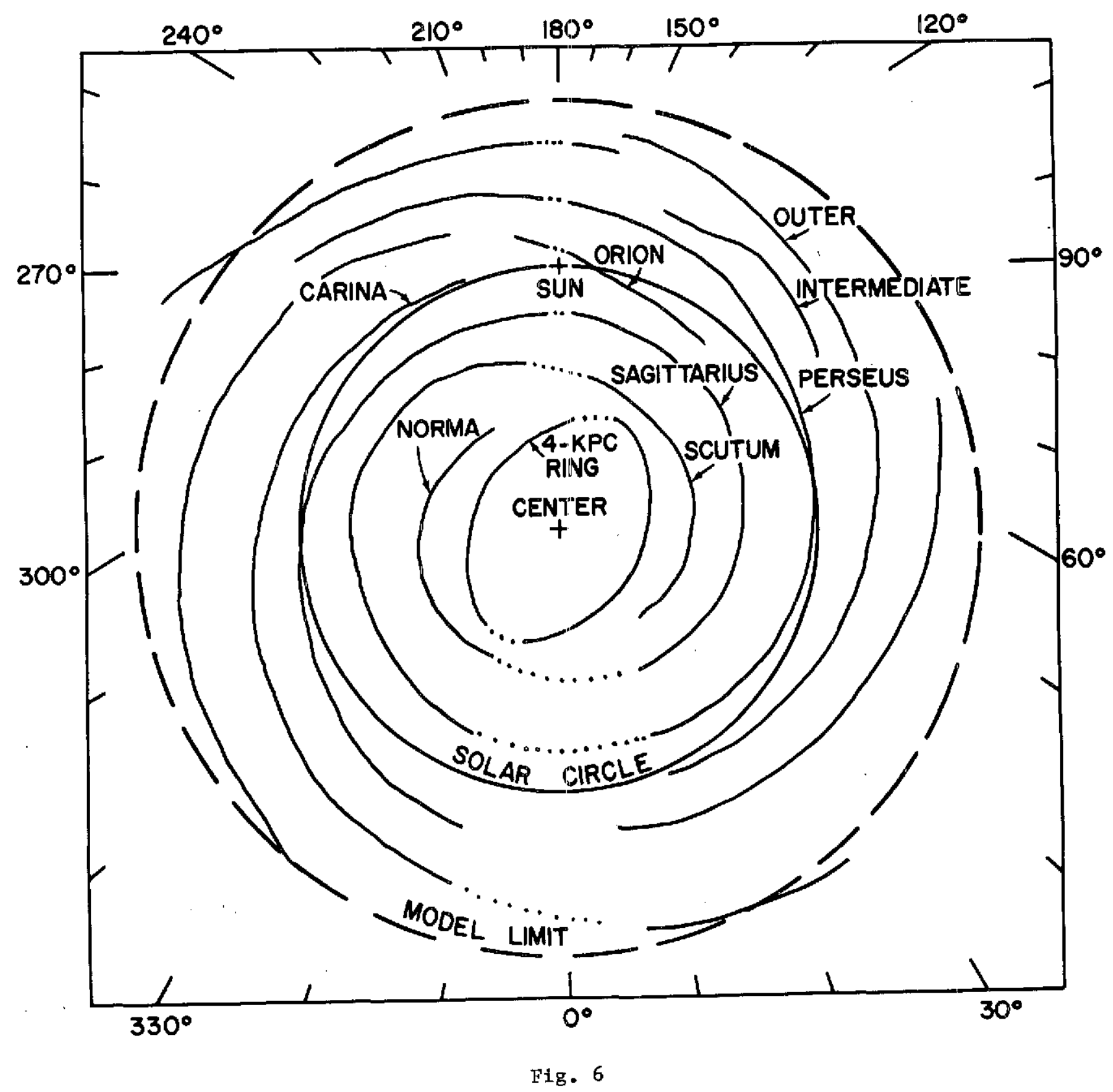




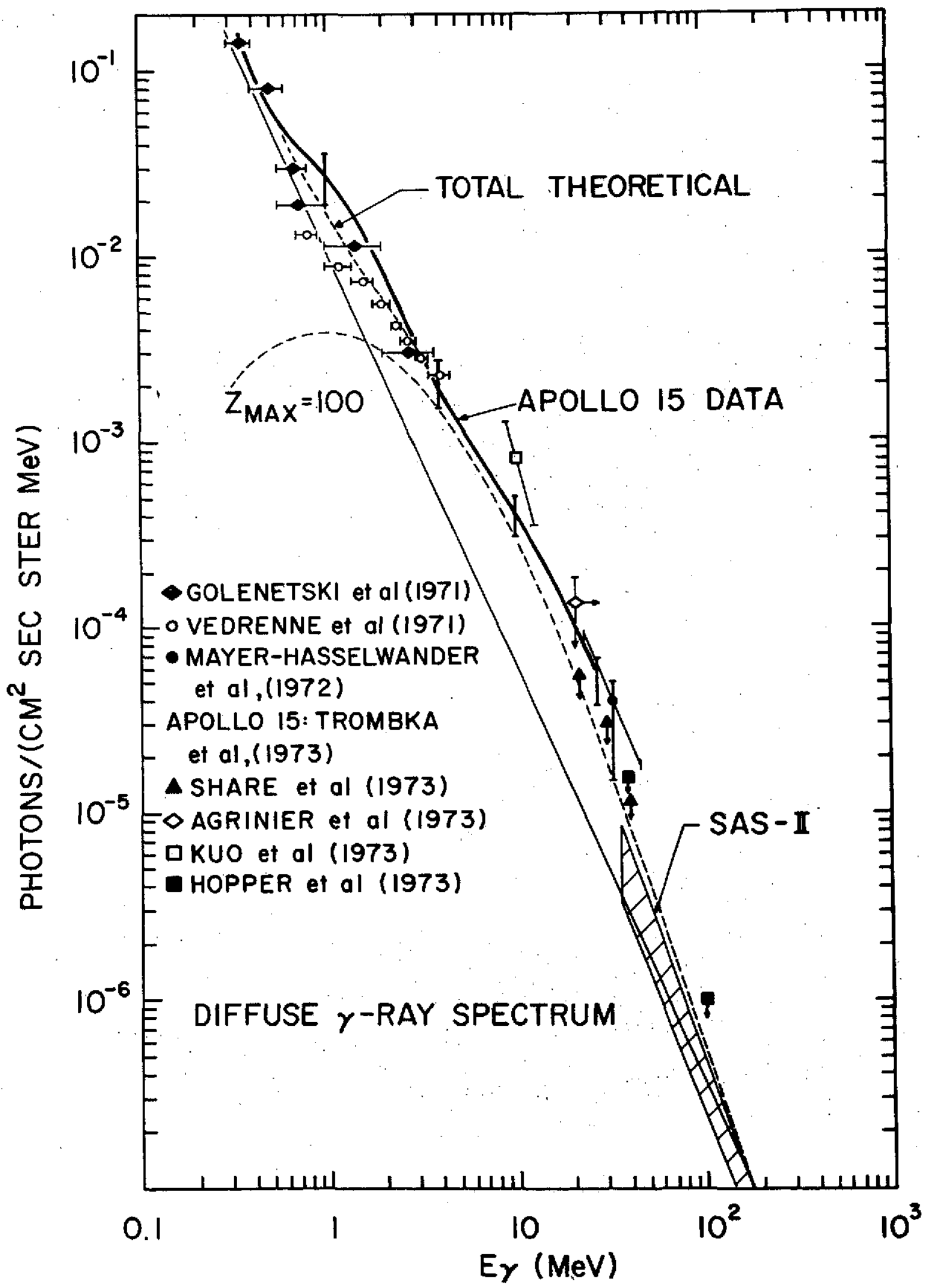

Fig. 7 

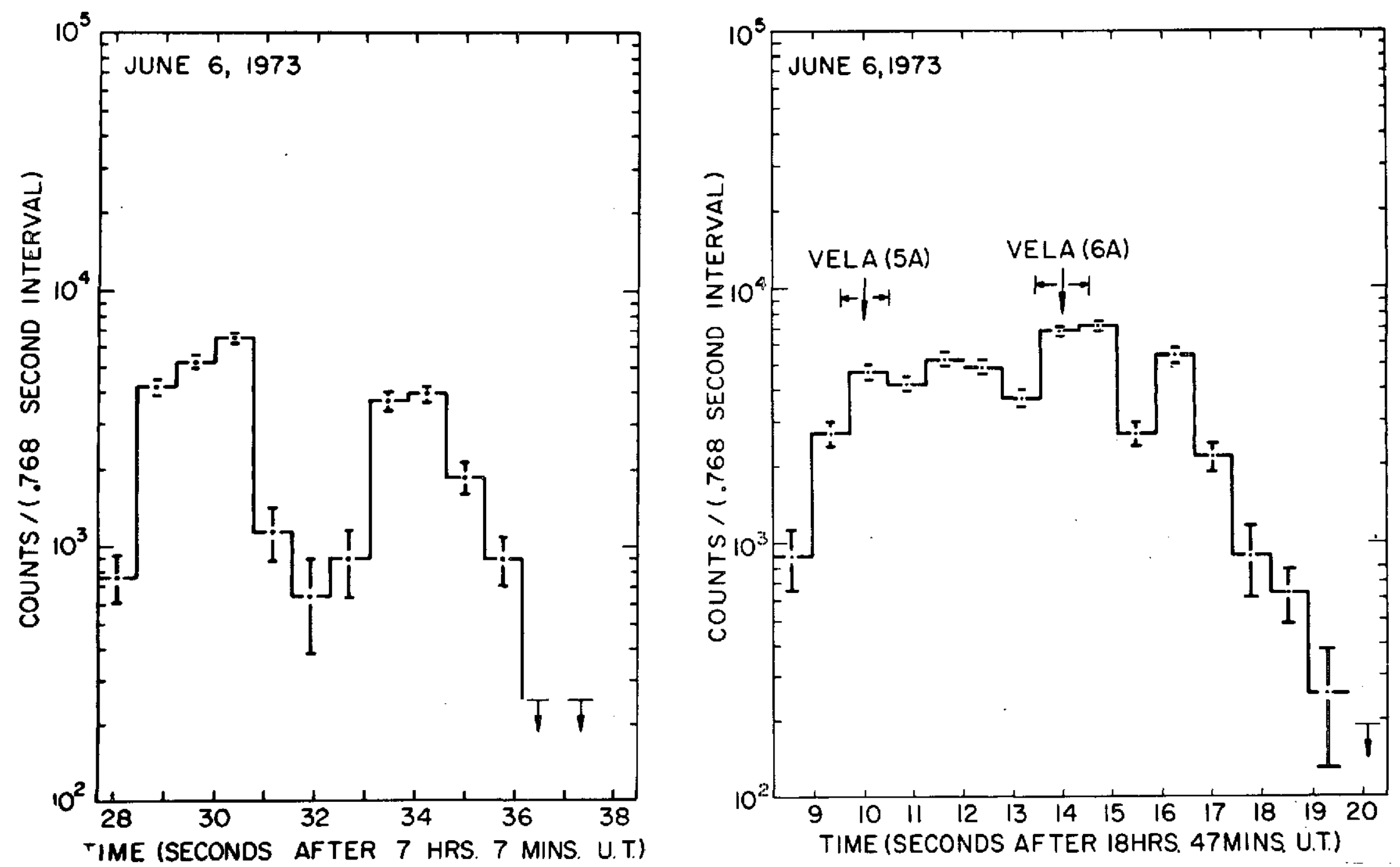

Fig. 8 


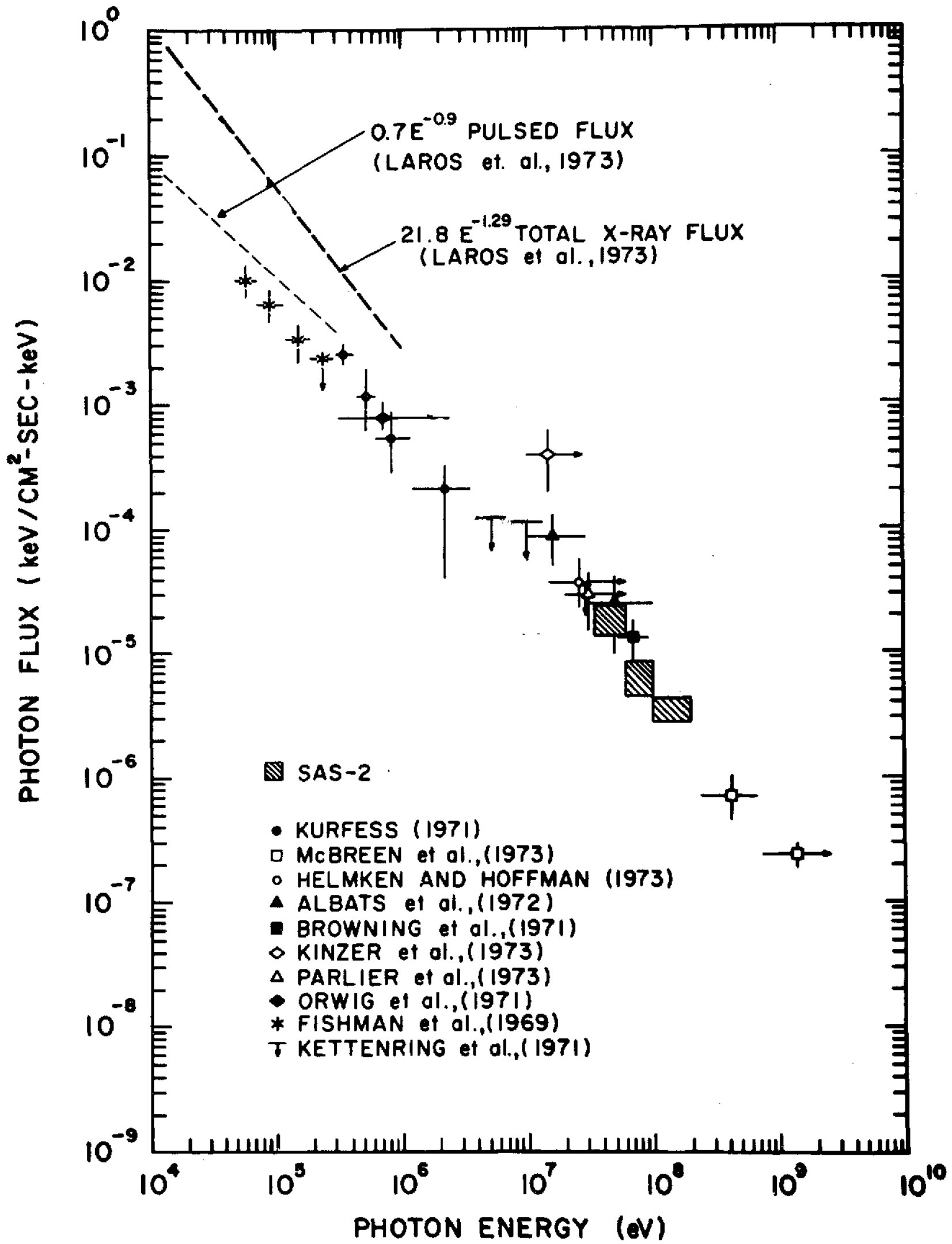

Fig. 9 